https://doi.org/10.15407/ujpe65.11.951

F.M. HUSSAIN,${ }^{1}$ F.A. MAJEED, ${ }^{1}$ M.H. METEAB ${ }^{2}$

${ }^{1}$ Department of Physics, College of Education for Pure Sciences, University of Babylon (Hillah, Iraq; e-mail: fouadattia@gmail.com)

2 Ministry of Education

(Hillah, Iraq)

\title{
INVESTIGATION OF THE CALCULATION OF COUPLED CHANNELS FOR SOME HALO SYSTEMS
}

\begin{abstract}
The fusion reaction for systems involving halo nuclei are investigated by two- and multicoupled channel calculations for the systems ${ }^{8} \mathrm{~B}+{ }^{58} \mathrm{Ni},{ }^{11} \mathrm{Be}+{ }^{209} \mathrm{Bi}$, and ${ }^{15} \mathrm{C}+{ }^{232} \mathrm{Th}$. The effect of coupling between the breakup channel and the elastic channel have been considered using the Continuum Discretized Coupled Channels (CDCC) method in full quantum and semiclassical approaches. The calculation of the fusion cross-section $\sigma_{\text {fus }}(\mathrm{mb})$, fusion barrier distribution $D_{\text {fus }}(\mathrm{mb} / \mathrm{MeV})$ and fusion probability $P_{\text {fus }}$ reproduces the measured data for the systems under study quite well above and below the Coulomb barrier $V_{B}$. In the case of two-channel coupling both in semiclassical and quantum mechanical approaches, the measured data above the Coulomb barrier $V_{B}$ are overestimated.

Keywords: one halo nuclei, weakly bound nuclei, breakup channel.
\end{abstract}

\section{Introduction}

The heavy-ion fusion is of essential significance in astro-physics also in the production of very heavy nuclei. Through the arrival of minor beams of nuclei reach in neutrons and protons, it is essential to evaluate the inclusive fusion of nuclear types as a duty of shelling energy specifically near the Coulomb barrier [1]. Minor beams are quickly creating a new domain of researches of the structure and reactions of unstable nuclei, specially of neutron-rich nuclei [2]. A number of new features of the structures have been discovered like the presence of a neutron halo near the neutron stable-line and the representative of low-energy summits in some nuclei, then, named soft dipole style, in the dynamism function of various many transitions $[2,3]$. Many of the neutron- and proton-rich nuclei reveal halo formations, with a built-in close core with one or more weakly bound nuclei filling faraway orbits [4].

(C) F.M. HUSSAIN, F.A. MAJEED, M.H. METEAB, 2020 ISSN 2071-0194. Ukr. J. Phys. 2020. Vol. 65, No. 11
The expanded investigation of the influence of breakup reactions is desired in order to create a trustworthy assess of the cross-section of fusion, due to a halo nucleus is assumed to be readily crashed [2]. The lengthy tail in the nuclear matter density is considered the astonishing advantage which is characterized by loosely bound halo nuclei. The prospect is maximum, when the nucleus is excited to continuum states in comparison with the stable nuclei. Furthermore, if the other reaction methods are used, the possible coupling is stronger $[5,6]$. When unstable nuclear beams will be more available at many laboratories, it will be possible to study the fusion mechanism of nuclei with important characteristics. The halo nuclei are weakly bound and have low separation energies, but the distributions of the nuclear density are largely extended and confine the momentum distribution [6]. The optical potential is the main element in fusion, and its role is fundamental in breakup calculations [5]. The halo itself characterizes an improvement at around the Coulomb barrier, and the split- 
up handicaps the fusion; the influence is considered more significant at the upper Coulomb barrier. Of course, we should remember that ${ }^{6} \mathrm{He}$ is a Borromean two-neutron halo, but ${ }^{11} \mathrm{Be}$ is a one-neutron halo nucleus. C. Signorini et al. [7, 8] obtained that the stable,tightly bound ${ }^{10} \mathrm{Be}$ isotope gives a bigger fusion cross-section than that of ${ }^{11} \mathrm{Be}$ at a subbarrier. We mention the comparison the ${ }^{6} \mathrm{He}$ and ${ }^{4} \mathrm{He}$ fusion with ${ }^{209} \mathrm{Bi}$ by J.J Kolata et al. [9], where the influences of the halo (augmentation) at subbarrier energies were very obvious. The results on the protonrich nuclei ${ }^{17} \mathrm{~F}$ fusion to ${ }^{208} \mathrm{~Pb}$ was determined in [10]. The ground state of this nucleus is be a normal state, but its first excited state is generally $l=0$ and seems to display halo characteristics [10]. Recently, F.A. Majeed and Y.A Abdul-Hussien [11] had studied the effect of the breakup channel for ${ }^{6,8} \mathrm{He}$ halo nuclei. Majeed et al. had performed semiclassical coupled-channels calculations in a heavyion fusion reaction for the systems ${ }^{40} \mathrm{Ar}+{ }^{110} \mathrm{Pd}$ and ${ }^{132} \mathrm{Sn}+{ }^{48} \mathrm{Ca}$. They proved that the semiclassical approach including the coupling between the elastic channel and the continuum proves to be very successful in describing the total fusion reaction crosssection $\sigma_{\text {fus }}$ and the fusion barrier distribution $D_{\text {fus }}$ below and above the Coulomb barrier for a medium and heavy systems [12]. The effect of the breakup channel on fusion reactions of weakly bound systems by means of the semiclassical and full quantum mechanical approaches has been discussed by F.A. Majeed [13]. F.A. Majeed et al. performed coupled-channel calculations using the semiclassical and full quantum mechanical calculations to study the effect of channel coupling on the calculations of the total fusion reaction cross-section $\sigma_{\text {fus }}$, the fusion barrier distribution $D_{\text {fus }}$, and the fusion probability $P_{f}$ for the light systems ${ }^{4} \mathrm{He}+{ }^{233} \mathrm{U},{ }^{13} \mathrm{C}+{ }^{48} \mathrm{Ti}$ and the medium system ${ }^{46} \mathrm{Ti}+{ }^{46} \mathrm{Ti}$. The effect of the breakup channel on the fusion reaction of weakly bound systems by means of a quantum-mechanical approach has been conducted by F.A. Majeed and F.A Mahdi [14]. They had concluded that, for halo systems, it is very important to include the breakup channel in the fusion reaction calculaitons.

In this study, the effect of the breakup channel has been conducted for the fusion reactions from cross-sections, distribution barrier, and probabilities in collisions of weakly bound halo nuclei. Our calculations are utilized the Continuum Discretized
Coupled-Channel (CDCC) method by the Alder and Winther (AW) theory to illustrate the coupled channels which are more complicated. The systems ${ }^{8} \mathrm{~B}+{ }^{58} \mathrm{Ni},{ }^{11} \mathrm{Be}+{ }^{209} \mathrm{Bi}$, and ${ }^{15} \mathrm{C}+{ }^{232} \mathrm{Th}$ are calculated by using the $\mathrm{CC}$ code for full quantum mechanics to compare with the SCF code in semiclassical mechanics; these codes were coded and established by H.D. Marta et al. [15].

\section{Theoretical Framework}

The theoretical framework of the tunneling phenomenon, like subbarrier energies, with the strong coupling of channels which is the Alder and Winther (AW) theory [16-18]. The coupled-channels method has been widely and efficaciously utilized to illustrate the fusion steady nuclei at a subbarrier, which shows augmentation over the single barrier state [1]. The coupling of channels is taken into account to match to bound states of the partners. The expansion of this theory to the fusion of unstable (halo) nuclei, which includes the coupling with the breakup channel was achieved is the past by using deuteron projectiles scattered on various targets in the Continuum Discretized Coupled Channels (CDCC) Method [19]. Involving the breakup channel within coupling is consider very difficult, since an infinite set of three or more nucleons in the continuum state should be described in the breakup channel. For empirical utilizes, it is required to describe the continuum by a finite set of states $[20,21]$. In the state of nuclei halo included one neutron, this can be achieved by the CDCC method. However, the enforcement of this process is more complex [18]. The representation of a coupled channel in fusion reactions, where the fusion cross-sections can be resulted from the total reaction cross-sections, looks as $[19,22]$.

$\sigma_{f}=\sigma_{R}-\sigma_{D}$

where $\sigma_{D}$ is the cross-section of the direct reaction, and $\sigma_{R}$ is given by

$\sigma_{R}=\frac{k}{E}\left\langle\psi_{j}^{(+)}|-\operatorname{Im} V| \psi_{j}^{(+)}\right\rangle$,

where $\left\langle r \mid \psi_{j}^{(+)}\right\rangle$is the wave function that describes the elastic scattering, and $V$ is the full potential [19,23],

$V(r)=V_{N}(r)+V_{C}(r)$,

ISSN 2071-0194. Ukr. J. Phys. 2020. Vol. 65, No. 11 
and

$\sigma_{f}=\frac{k}{E} \sum_{k}\left\langle\psi_{j k}^{(+)}\left|-\operatorname{Im} V_{k}\right| \psi_{j k}^{(+)}\right\rangle$,

where $V_{k}$; is the optical potential in the channel $k$ (single channel), and $\left|\psi_{j k}^{(+)}\right\rangle$is the wave function of the exact scattering in the same channel. Equation (4) has been utilized by many authors to calculate $\sigma_{f}$ utilizing programs with coupled channels [16]. Other models centered on Eq. (4), but with the more supposition of infinite absorption, when the barrier is penetrable, have also been advanced [8]. The models of the second rank are popularized by fusing the influence breakup channel (involved in $\left|\psi_{j k}^{(+)}\right\rangle$). To include the breakup channel coupling influence, in the crosssection equation, specifically, the nonzero width of the excited state, it is suitable first to definite the crosssection as a sum of partial-wave contributions $[22,24]$

$\sigma_{f}=\frac{\pi}{k^{2}} \sum_{l=0}^{\infty}(2 l+1)\left(1-T_{l}^{B}\right) \times$

$\times \frac{1}{2}\left[T_{l}^{f}\left(E_{l}+G\right)+T_{l}^{f}\left(E_{l}-G\right)\right]$,

where the $+(-)$ sign shows additive (subtraction) to (from) the barrier height, and $\left(1-T_{l}^{B}\right)$ is the breakup survival probability given as [24]

$1-T_{l}^{B}=\exp \left[\frac{-4 \mathfrak{F}_{0}^{2} \pi}{E_{l}^{2}}\left|S_{l}^{(1)}\right| I_{l}^{(1)}(\eta, S)\right]$,

where $S_{l}^{(1)}$ is the modulus of the elastic $S$ matrix in the breakup channel, $\mathfrak{F}_{0}^{2}$ is a coupling strength factor, and $I_{l}^{(1)}(\eta, S)$ is a Coulomb radial integral as the transmission factor $T_{l}^{f}$ was approximated by the HillWheeler formula:

$T_{l}^{f}=\left\{1+\exp \left[\frac{-2 \pi}{\hbar \omega}\left[V_{B}+\frac{\hbar l(l+1)}{2 \mu R_{B}^{2}}-E_{l}\right]\right]\right\}^{-1}$,

where $V_{B}, R_{B}$, and $\hbar \omega$ are the optical potential barrier parameters, giving its height, radius, and curvature, respectively.

The theoretical thought and the empirical investigation of the state of a distortion nucleus, as the classical shape of a distorted body oriented in various directions in the space is convenient. It is simple to assessed the presence of a distributions of fusion barriers [16]; an empirical barrier distribution can be found from the measured data by the relation;

$\operatorname{Dis}_{\exp }(E)=\frac{d^{2}(E \sigma)}{d E^{2}}$,

where $E$ is defined as the incident energy, and $\sigma$ is the measured cross-sections of fusion. This requires finding a second derivative of the data, which oblige very intensive restrictions on their accuracy. The current review confirms those data that are capable to happen these accuracy requirements $[16,25]$.

\section{Results and Discussion}

In order to consider the breakup channel in the coupling calculations, the fusion reactions for one-proton and one-neutron halo nuclei are studied by adopting the CDCC method, to calculate the cross-section $\sigma_{\text {fus }}$, distribution of barriers $D_{\text {fus }}$, and probabilities of barriers fusion $P_{\text {fus }}$. The diagonal elements of the matrix of the interaction potential in the $\mathrm{CC}$ code were taken to give the real and imaginary parts of the Woods-Saxon potential, each deepness, radius, and diffuseness. This results from the fit to the empirical Coulomb barrier with height $V_{B}$ at $R_{B}$ to match the shape of the calculated barrier from the AküzWinther (AW) potential as tabulated in Table.

In all the proceeding figures, the calculations of the fusion cross-sections $\sigma_{\text {fus }}(\mathrm{mb})$, fusion barrier distribution $D_{\text {fus }}(\mathrm{mb} / \mathrm{MeV})$, and probability of fusion barrier penetration $P_{\text {fus }}$ are represented by solid and dashed (red and blue) curves, using the multichannel coupling for the semiclassical and full quantum mechanical results, respectively. The solid (green and purple) curves represent the calculations using twochannel coupling only for the calculations of the fusion cross-section $\sigma_{\text {fus }}$ for the semiclassical and full quantum mechanical approaches, respectively. The codes used in the present work have coded names $(S C F)$ for semiclassical calculations and $(C C)$ for quantum mechanical calculations. The blue arrow on the $x$-axis represents the position of the Coulomb barrier.

\section{1. ${ }^{8} \mathrm{~B}+{ }^{58} \mathrm{Ni}$}

The calculations of the total fusion cross-section $\sigma_{\text {fus }}$, the fusion barrier distribution $D_{\text {fus }}$, and the fusion probability $P_{\text {fus }}$ are shown in Fig. 1, panel A, panel 

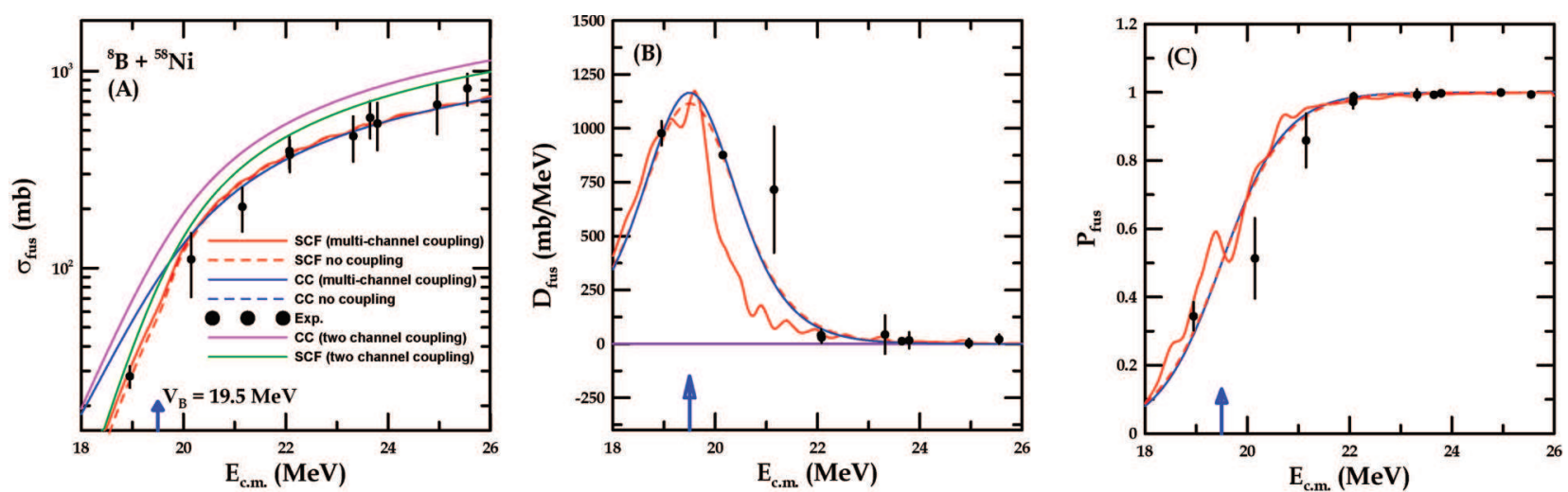

Fig. 1. Comparison of the excitation function calculations of the cross-sections $\sigma_{\text {fus }}(\mathrm{mb})$, barrier distributions $D_{\text {fus }}(\mathrm{mb} / \mathrm{MeV})$, and probability $P_{\text {fus }}$ in the semiclassical mechanical $S C F$ code (red curves) and the full quantum mechanical $C C$ code (blue curves) with the measured data (solid circles) [26] for the ${ }^{8} \mathrm{~B}+{ }^{58} \mathrm{Ni}$ reaction

The parameters of the AW potential with the associated

Coulomb barrier height for the systems ${ }^{8} \mathrm{~B}+{ }^{58} \mathrm{Ni},{ }^{11} \mathrm{Be}+{ }^{209} \mathrm{Bi}$ and ${ }^{15} \mathrm{C}+{ }^{232} \mathrm{Th}$

\begin{tabular}{|c|c|c|c|c|c|c|c|c|}
\hline Systems & $V_{0}(\mathrm{MeV})$ & $r_{0}(\mathrm{fm})$ & $a_{0}(\mathrm{fm})$ & $W_{i}(\mathrm{MeV})$ & $r_{i}(\mathrm{fm})$ & $a_{i}(\mathrm{fm})$ & \multicolumn{2}{|l|}{$\begin{array}{c}V_{B} \\
(\mathrm{MeV})\end{array}$} \\
\hline${ }^{8} \mathrm{~B}+{ }^{58} \mathrm{Ni}$ & 76.2 & 1.20 & 0.7 & 25.4 & 0.935 & 0.779 & 19.50 [Present work] & $20[26]$ \\
\hline${ }^{11} \mathrm{Be}+{ }^{209} \mathrm{Bi}$ & 214.4 & 1.01 & 0.88 & 71.5 & 0.952 & 0.769 & 37.50 [Present work] & $38[27]$ \\
\hline${ }^{15} \mathrm{C}+{ }^{232} \mathrm{Th}$ & 163.8 & 1.20 & 0.60 & 54.6 & 0.970 & 0.758 & $59.0[28]$ & \\
\hline
\end{tabular}

B, and panel C, respectively. The set of Woods-Saxon parameters used are tabulated in Table. The calculation for $\sigma_{\text {fus }}$ both for two-channel and single-channel for both semiclassical and quantum mechanical approaches in the case of coupling and without coupling are presented in panel A of Fig. 1. It is clear that the two-channel approach overestimated the measured data. The multichannel coupling for both semiclassical and quantum mechanical is able to reproduce the experimental data. In order to find the best calculations in comparison with the measured data, the $\chi^{2}$ method has been adopted. The $\chi^{2}$ values obtained are the best and the closet to measured data in both codes $\chi^{2}=0.00387823,0.00560114$ and $\chi^{2}=$ $=0.11406700,0.12125910$, which corresponds to the calculations including no coupling and coupling channels for SCF below and above the Coulomb barrier $\left(V_{B}\right)$, respectively, are in the best agreement with the experimental data from single and coupling channel for $C C$ calculations at values $\chi^{2}=0.1$, 0.11607760 for below and above $V_{B}$. The multichannel coupling in both $S C F$ and $C C$ codes of the coupling that included the obtained $\chi^{2}$ values are $\chi^{2}=0.03477085,0.37931930$ and $\chi^{2}=0.00633482$, 0.18632040 . The value of $\chi^{2}=0.00849025$ for the fusion probability $P_{\text {fus }}$ below the Coulomb barrier, while, for the fusion barrier distribution $D_{\text {fus }}$, the coupled channel value is better in $S C F$ calculations with $\chi^{2}=0.00003272$. For calculations obtained above the Coulomb barrier for $D_{\text {fus }}, P_{\text {fus }}$ to be in the best agreement with data that are $\chi^{2}=0.05917057,0.09160499$ in a single-channel in $S C F$. The $S C F$ calculations are in the best and a good agreement with $C C$ calculations of the data for this reaction.

\section{2. ${ }^{11} \mathrm{Be}+{ }^{209} \mathrm{Bi}$}

This reaction appears to include the very necessary breakup channel with the elastic channel in breakup processes of ${ }^{11} \mathrm{Be}$ halo nucleus. The coupling multi carve to the breakup channel is required to enhance the results around the barrier. The experimental data for this reaction are taken from [27] and shown in Fig. 2, panel A for the comparison. The potential parameters of this reaction are presented in Table. The value of the barrier height in the present

ISSN 2071-0194. Ukr. J. Phys. 2020. Vol. 65, No. 11 

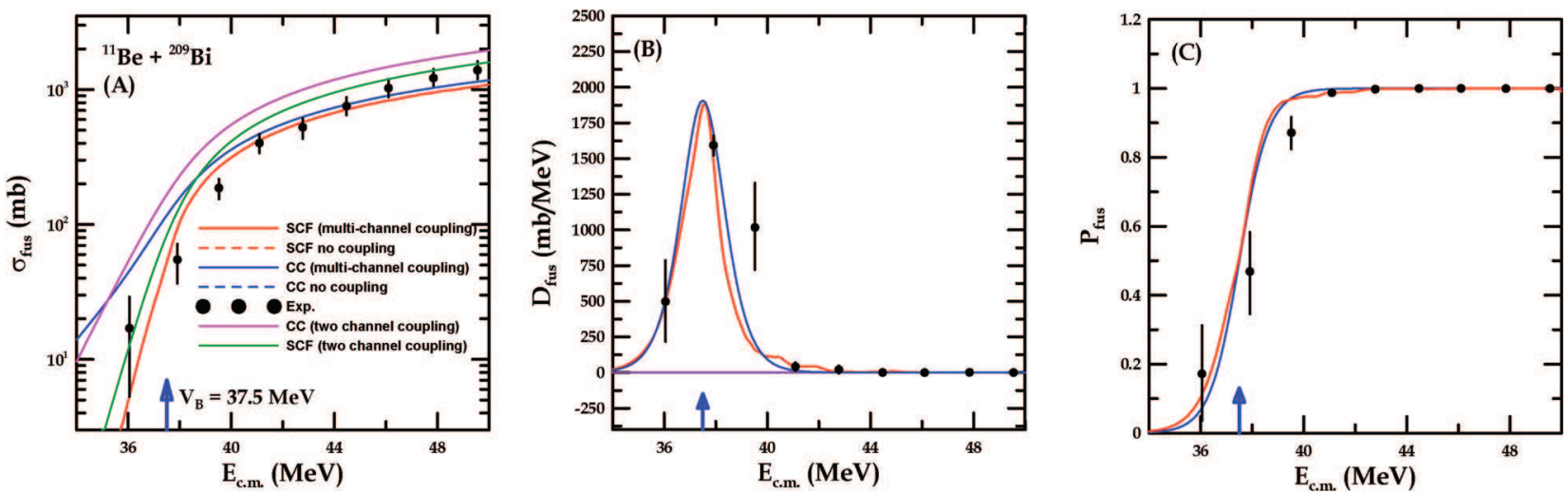

Fig. 2. Comparison of the excitation function calculations of the cross-sections $\sigma_{\text {fus }}$ (mb), barrier distributions $D_{\text {fus }}$ (mb/MeV), and probability $P_{\text {fus }}$ in the semiclassical mechanical $S C F$ code (red curves) and the full quantum mechanical $C C$ code (blue curves) with the measured data (solid circles) [27] for ${ }^{11} \mathrm{Be}+{ }^{209} \mathrm{Bi}$ reaction
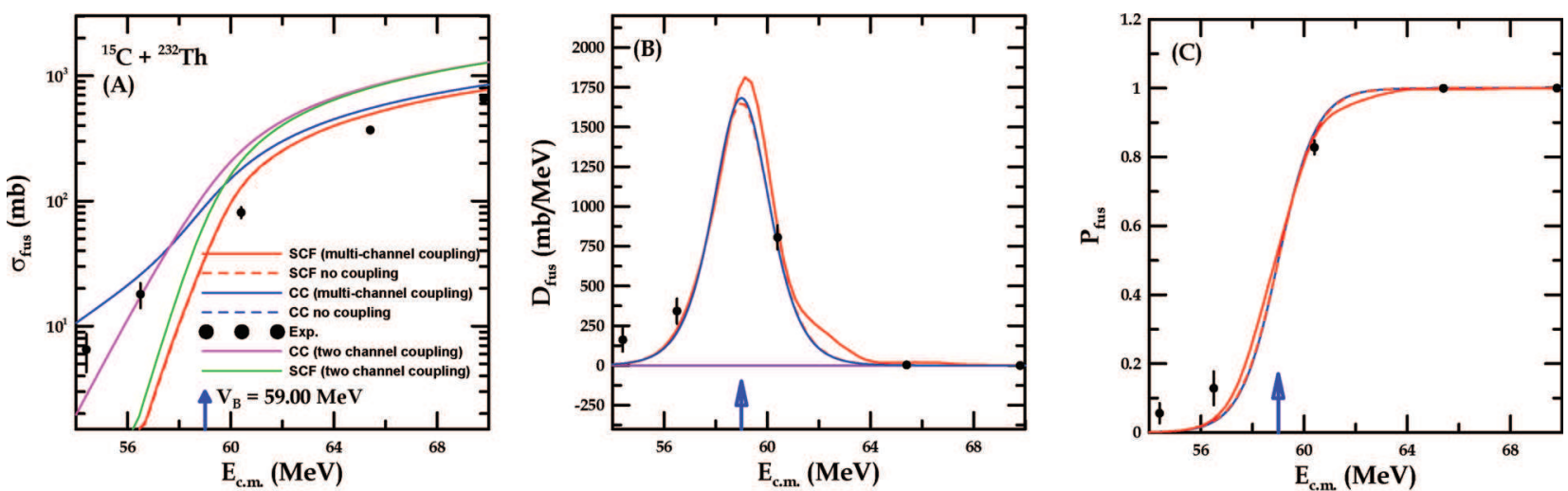

Fig. 3. Comparison of the excitation function calculations of the cross-sections $\sigma_{\text {fus }}(\mathrm{mb})$, barrier distributions $D_{\text {fus }}$ (mb/MeV), and probability $P_{\text {fus }}$ in the semiclassical mechanical $S C F$ code (red curves) and the full quantum mechanical $C C$ code (blue curves) with the measured data (solid circles) [28] for ${ }^{15} \mathrm{C}+{ }^{232} \mathrm{Th}$ reaction

work is shifted down by $0.5 \mathrm{MeV}$ from the value $20 \mathrm{MeV}$ in Ref. [27]. The best Chi-square value is $\chi^{2}=0.06776251$, which corresponds to calculations with the single channel and coupled channels in best agreements with the measured data for the crosssection of total fusion $\sigma_{\text {fus }}$ in $C C$ under barrier for the multi-carve coupling, while $\chi^{2}=0.11111110$ in the two-carve coupling. As for the $S C F$ value for the two-carve coupling, the best $\chi^{2}=0.03700209$. Above the barrier, we found a good agreement with data at $\chi^{2}=0.02204157$ in the SCF code. The calculations of $\sigma_{\text {fus }}$ for the multi-carve coupling are enhanced in both semiclassical and quantum mechanical approaces. The results for the barrier distribution $D_{\text {fus }}$ are presented in panel $\mathrm{B}$, the least value is $\chi^{2}=0.00012620$ in one and coupling barrier that is in the perfectly convention with the empirical data

ISSN 2071-0194. Ukr. J. Phys. 2020. Vol. 65, No. 11 in $C C$ calculations under the Coulomb barrier and the closer compared with one and coupled channel cases to $S C F$ is $\chi^{2}=0.00013403$, respectively. The calculated fusion probability $P_{\text {fus }}$ is shown in panel C. The better value is $\chi^{2}=0.03559827,0.03187320$ in the coupled case for $S C F$ and $C C$ codes above the barrier.

\section{3. ${ }^{15} C+{ }^{232} T h$}

To describe the fusion processes of one-neutron halo nuclei by analysis of the fusion excitation functions for the cross-section, distribution, and probability as shown in Fig. 3, panels A, B, and C. The agreement of the measured cross-section $\sigma_{\text {fus }}$ with the predicted one with coupledc channels for this reaction has been achieved in both semiclassical and quantum treatment. The results of calculations for the multi- 
channel coupling and the coupling of two channels were compared with the measured data for this reaction taken from Ref. [28]. The Woods-Saxon parameters used in the present calculations are displayed in Table. The best value of theoretical calculations for $\sigma_{\text {fus }}$ is found at $\chi^{2}=0.00042131$ for the semiclassical approach and at $\chi^{2}=0.00104671$ for the full quantum mechanical one. The account for many fusion channels significantly improves calculations up to the barrier, while the calculations with two fusion channels cannot support calculations well. The values are $\chi^{2}=0.00121761,0.00171838$, and are $\chi^{2}=0.00022444,0.00013053$, in CC and SCF for $D_{\text {fus }}$ and $P_{\text {fus }}$ to excellent agreement with experimental data above barrier. In CC and SCF codes, all calculations are nearly the empirical data above barrier for this reaction.

\section{Conclusion}

The present investigation shows clearly that the twochannel approach in both semiclassical and quantum mechanical calculations were unable to describe the measured data, even when the coupling is included especially above the Coulomb barrier. The multichannel coupling calculations are in better agreement with the corresponding experimental data. The fusion barrier distribution and fusion probability have ripple and unstable behaviour in the coupled channel calculations. The coupled channel should be considered for all the calculations, and this in agreement with our previous work and with other works.

1. C.A. Bertulani, M.S. Hussein, G. Münzenberg. Physics of Radioactive Beams (Nova Science, 2001).

2. N. Takigawa, M. Kuratani, H. Sagawa. Effect of breakup reactions on the fusion of a halo nucleus. Phys. Rev. C 47, 2470 (1993).

3. R.N. Boyd, I. Tanihata. Physics with radioactive nuclear beams. Phys. Today 4544 (1992).

4. S. Nakayama, T. Yamagata, H. Akimune, I. Daito, H. Fujimura, Y. Fujita, M. Fujiwara, K. Fushimi, T. Inomata, H. Kohri, N. Koori, K. Takahisa, A. Tamii, M. Tanaka, H. Toyokawa. Soft dipole resonance in the neutron-skin nucleus ${ }^{6}$ He. Phys. Rev. Lett. 85, 262 (2000).

5. M.Y.M. Hassan, M.Y.H. Farag, E.H. Esmael, H.M. Maridi. Elastic scattering and breakup effect analysis of ${ }^{11} \mathrm{Be}+{ }^{12} \mathrm{C}$ at $38.4 \mathrm{MeV} /$ nucleon. Phys. Rev. C 79, 064608 (2009).

6. R. Kumari. On the fusion probabilities of halo nuclei. Proceedings of the DAE Symp. Nucl. Phys. 58, 340 (2013).

7. C. SignoriniZ, H. LiuZ, C. LiK, E.G. Löbner, L. Müller, M. Ruan, K. Rudolph, F. Soramel, C. Zotti, A. An- drighetto, L. Stroe, A. Vitturi, H.Q. Zhang. Does break-up affect ${ }^{9} \mathrm{Be}+{ }^{209} \mathrm{Bi}$ fusion at the barrier. Eur. Phys. J. A 5, 7 (1999).

8. C. Signorini. Interaction at the barrier in the systems ${ }_{9,10,11} \mathrm{Be}+{ }^{209} \mathrm{Bi}$ : Well-established facts and open questions. Eur. Phys. J. A 13, 129 (2002).

9. J.J. Kolata, V. GuimarГJes, D. Peterson, P. Santi, R. White-Stevens, P.A. DeYoung, G.F. Peaslee, B. Hughey, B. Atalla, M. Kern, P.L. Jolivette, J.A. Zimmerman, M.Y. Lee, F.D. Becchetti, E.F. Aguilera, E. MartinezQuiroz, J.D. Hinnefeld. Sub-barrier fusion of ${ }^{6} \mathrm{He}$ with ${ }^{209}$ Bi. Phys. Rev. Lett. 81, 4580 (1998).

10. K.E. Rehm, H. Esbensen, C.L. Jiang, B.B. Back, F. Borasi, B. Harss, R.V.F. Janssens, V. Nanal, J. Nolen, R.C. Pardo, M. Paul, P. Reiter, R.E. Segel, A. Sonzogni, J. Uusitalo, A.H. Wuosmaa. Fusion cross sections for the proton drip line nucleus ${ }^{17} \mathrm{~F}$ at energies below the Coulomb barrier. Phys. Rev. Lett. 81, 3341 (1998).

11. F.A. Majeed, Y.A. Abdul-Hussien. Semiclassical treatment of fusion and breakup processes of ${ }^{6 ; 8} \mathrm{He}$ halo nuclei. J. Theo. Appl. Phys. 10, 107 (2016).

12. F.A. Majeed, R.S. Hamodi, F.M. Hussain. Effect of coupled channels on semiclassical and quantum mechanical calculations for heavy ion fusion reactions. J. Comp. and Theo. Nanosci. 14, 2242 (2017).

13. F.A. Majeed. The role of the breakup channel on the fusion reaction of light and weakly bound nuclei. Int. J. Nucl. Energ. Sci. Tech. 11, 218 (2017).

14. F.A. Majeed, F.A. Mahdi. Quantum mechanical calculations of a fusion reaction for some selected halo systems. Ukr. J. Phys. 64, 11 (2019).

15. H.D. Marta, L.F. Canto, R. Donangelo, P. Lotti. Validity of the semiclassical approximation for the breakup of weakly bound nuclei. Phys. Rev. C 66, 024605 (2002).

16. M. Dasgupta, D.J. Hinde, N. Rowley, A.M. Stefanini. Measuring barriers to fusion. Annu. Rev. Nucl. Part. Sci. 48, 401 (1998).

17. G.R. Satchler, M.A. Nagarajan, J.S. Lilley, I.J. Thompson. Heavy-ion fusion: Channel-coupling effects, the barrier penetration model, and the threshold anomaly for heavyion potentials. Ann. Phys. 178, 110 (1987).

18. A.M.S. Breitschaft, V.C. Barbosa, L.F. Canto, M.S. Hussein, E.J. Moniz, J. Christley, I.J. Thompson. Coupledchannel effects on heavy-ion sub-barrier fusion within the doorway expansion method. Ann. Phys. 243, 420 (1995).

19. W.H.Z. Cárdenas, L.F. Canto, R. Donangelo, N. Carlin, M.S. Hussein. Schematic coupled-channel calculations for nuclear reactions with unstable beams. Braz. J. Phys. 34, 1254 (2004)

20. K. Hagino, A. Vitturi, C.H. Dasso, S.M. Lenzi. Role of breakup processes in fusion enhancement of drip-line nuclei at energies below the Coulomb barrier. Phys. Rev. C 61, 037602 (2000).

21. A. Diaz-Torres, I.J. Thompson. Effect of continuum couplings in fusion of halo ${ }^{1} 1 \mathrm{Be}$ on ${ }^{208} \mathrm{~Pb}$ around the Coulomb barrier. Phys. Rev. C 65, 024606 (2002).

ISSN 2071-0194. Ukr. J. Phys. 2020. Vol. 65, No. 11 
22. M.S. Hussein, M.P. Pato, L.F. Canto, R. Donangelo. Nearbarrier fusion of ${ }^{11} \mathrm{Li}$ with heavy spherical and deformed targets. Phys. Rev. C 46, 377 (1992).

23. A. Kumari, R. Kharab. Role of projectile breakup in ${ }^{6} \mathrm{He}$ and ${ }^{6} \mathrm{Li}$ induced fusion reactions around barrier energies. Nucl. Phys. A 941, 38 (2015).

24. M.S. Hussein, M.P. Pato, L.F. Canto, R. Donangelo. Real part of the polarization potential for ${ }^{11} \mathrm{Li}$ induced fusion reactions. Phys. Rev. C 47, 2398 (1993).

25. N. Rowley, G. Satchler, P. Stelson. On the "distribution of barriers" interpretation of heavy-ion fusion. Phys. Lett. B 254, 25 (1991).

26. E.F. Aguilera, P. Amador-Valenzuela, E. Martinez-Quiroz, D. Lizcano, P. Rosales, H. García-Martínez, A. GómezCamacho, J.J. Kolata, A. Roberts, L.O. Lamm, G. Rogachev, V. Guimarães, F.D. Becchetti, A. Villano, M. Ojaruega, M. Febbraro, Y. Chen, H. Jiang, P.A. DeYoung, G.F. Peaslee, C. Guess, U. Khadka, J. Brown, J.D. Hinnefeld, L. Acosta, E.S. Rossi Jr, J.F.P. Huiza, T.L. Belyaeva. Near-barrier fusion of the ${ }^{8} \mathrm{~B}+{ }^{58} \mathrm{Ni}$ protonhalo system. Phys. Rev. Lett. 107, 092701 (2011).

27. M. Mazzocco, C. Signorini, M. Romoli, A. De Francesco, M. Di Pietro, E. Vardaci, K. Yoshida, A. Yoshida, R. Bonetti, A. De Rosa, T. Glodariu, A. Guglielmetti, G. Inglima, M. La Commara, B. Martin, D. Pierroutsakou, M. Sandoli, F. Soramel, L. Stroe, R. Kanungo, N. Khai, T. Motobayashi, T. Nomura, T. Ishikawa, H. Ishiyama, S. Jeong, H. Miyatake, M.H. Tanaka, I. Sugai, Y. Watan- abe. Scattering of ${ }^{11} \mathrm{Be}$ halo nucleus from ${ }^{209} \mathrm{Bi}$ at the Coulomb barrier. Eur. Phys. J. A 28, 295 (2006).

28. M. Alcorta, K.E. Rehm, B.B. Back, S. Bedoor, P.F. Bertone, C.M. Deibel, B. DiGiovine, H. Esbensen, J.P. Greene, C.R. Hoffman, C.L. Jiang, J.C. Lighthall, S.T. Marley, R.C. Pardo, M. Paul, A. Rogers, C. Ugalde, A.H. Wuosmaa. Fusion reactions with the one-neutron halo nucleus ${ }^{15}$ C. Phys. Rev. Lett. 106, 172701 (2011).

Received 29.07.19

Ф.М. Хуссейн, Ф.А. Маджид, М.Х. Метіб

АНАЛІЗ РОЗРАХУНКІВ ПОВ'ЯЗАНИХ КАНАЛІВ ДЛЯ ДЕЯКИХ ГАЛО СИСТЕМ

$\mathrm{P}$ е $з$ ю м е

Розглядаються реакції синтезу в системах з гало ядрами ${ }^{8} \mathrm{~B}+{ }^{58} \mathrm{Ni},{ }^{11} \mathrm{Be}+{ }^{209} \mathrm{Bi} \mathrm{i}{ }^{15} \mathrm{C}+{ }^{232} \mathrm{Th}$ у випадках зв'язку двох i багатьох каналів. Ефект зв'язку каналів розпаду і пружного розсіювання вивчено методом континуально-дискретних пов'язаних каналів у повністю квантовому і напівкласичному підходах. Результати розрахунків перерізу синтезу $\sigma_{\text {fus }}$ (мб), розподілу бар'єра синтезу $D_{\text {fus }}$ (мб/MeB) і ймовірності синтезу $P_{\text {fus }}$ добре описують експериментальні дані для досліджуваних систем вище і нижче кулонівського бар'єра $V_{B}$. У разі зв'язку двох каналів як для напівкласичного, так і для квантового підходів результати вище кулонівського бар'єра $V_{B}$ виявляються переоціненими. 